\title{
Prolonged inhibition of class I PI3K promotes liver cancer stem cell expansion by augmenting SGK3/GSK-3 $\beta / \beta$-catenin signalling
}

Fengchao Liu, Xiaoling Wu, Xin Jiang, Yanzhi Qian and Jian Gao*

\begin{abstract}
Background: Serum and glucocorticoid-regulated kinase 3 (SGK3) has been reported to play an important role in tumour progression, but its role in cancer stem cells (CSCS) remains obscure. The phosphoinositide 3-kinase (PI3K) pathway is considered a hallmark of cancer. Although many PI3K pathway-targeted therapies have been tested in oncology trials, the results are not satisfactory.

Methods: We used spheroids cultured in serum-free culture medium and MicroBead isolation to obtain liver CSCs. Spheroid formation assay and flow cytometric analysis were performed to investigate liver CSC expansion. Real-time polymerase chain reaction (PCR), western blot and immunofluorescence were used to assess gene expression in cell lines.

Results: We found that SGK3 is preferentially activated in liver CSCs. Upregulated SGK3 significantly increases the expansion of liver CSCs. Conversely, suppression of SGK3 in human hepatocarcinoma (HCC) cells had an opposite effect. Mechanistically, SGK3 promoted $\beta$-catenin accumulation by suppressing GSK-3 $\beta$-mediated $\beta$-catenin degradation in liver CSCs, and then promoting the expansion of liver CSCs. Prolonged treatment of HCC cells with class I PI3K inhibitors leads to activation of SGK3 and expansion of liver CSCs. Inhibition of hVps34 can block SGK3 activity and suppress liver CSC expansion induced by PI3K inhibitors. More importantly, we also found that prolonged treatment of HCC cells with PI3K inhibitors stimulates the $\beta$-catenin signalling pathway via activation of SGK3.

Conclusions: Prolonged inhibition of class I PI3K promotes liver CSC expansion by augmenting SGK3-dependent $\beta$-catenin stabilisation, and effective inhibition of SGK3 signalling may be useful in eliminating liver CSCS and in PI3K pathway-targeted cancer therapies.
\end{abstract}

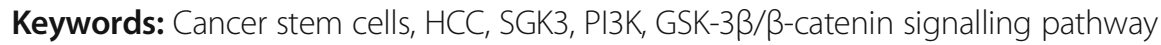

\section{Background}

Hepatocellular carcinoma (HCC) is one of the leading causes of cancer-related death and is the main severe consequence leading to death in patients with cirrhosis and many other chronic liver diseases [1,2]. Despite recent progress in $\mathrm{HCC}$ treatment, prognosis for this refractory disease remains unsatisfactory [3] because both solid tumours show considerable histological and functional heterogeneity [4]. Such cellular heterogeneity is very

\footnotetext{
* Correspondence: 982213482@qq.com

Department of Gastroenterology, Second Affiliated Hospital, Chongqing Medical University, Chongqing, China
}

important due to its important role in treatment resistance. Recent studies have suggested that subpopulations of cells with increased tumorigenesis capacities and self-renewal potential, termed as cancer stem cells (CSCs) [5], exist within tumours. Persistence of CSCs is a primary cause of relapse and metastasis, which are highly resistant to chemotherapy [6]. Therefore, more effective therapeutic strategies may be developed if the molecular mechanism underlying CSC regulation is illuminated.

The existence of CSCs has been demonstrated in a variety of solid tumours, including liver cancer [7]. Liver CSCs can be enriched with several defined surface

(c) The Author(s). 2018 Open Access This article is distributed under the terms of the Creative Commons Attribution 4.0 International License (http://creativecommons.org/licenses/by/4.0/), which permits unrestricted use, distribution, and 
markers, including CD133, CD90, CD44, OV6, EpCAM, CD13, CD24, ICAM-1, CD47, Lgr5, and keratin19 [8]. Although CSCs can be identified within the liver cancer cells, they cannot be effectively eradicated because the detailed regulatory mechanism of CSC generation and expansion remains largely unknown. Signalling pathways such as the Wnt/ $\beta$-catenin, TGF $\beta$, IL-6/STAT3, Notch and ANXA3/JNK pathways have been reported to be involved in the regulation of liver CSCs [9-12]. Among these pathways, Wnt/ $\beta$-catenin signalling has received increasing attention because of its important role in both normal stem cells and CSCs. Inhibition of the Wnt/ $/$-catenin pathway has also been shown to be effective in eliminating CSCs [13]. However, the deregulation of $\mathrm{Wnt} / \beta$-catenin pathway in liver CSCs is not fully understood.

The phosphoinositide 3-kinase (PI3K) pathway is a very important intracellular signalling pathway, which plays crucial roles in normal cell processes and a critical role in cancers. Several studies have explored the therapeutic targeting of the PI3K pathway in cancers, and various inhibitors targeting PI3K and its isoforms have been developed [14]; however, the clinical effect was not satisfactory. The role of the PI3K signalling pathway in CSCs has been reported, but some controversy remains [15].

Serum and glucocorticoid-regulated kinase 3 (SGK3), an AGC protein kinase family member, has been found to play a critical role in a variety of cancers [16]. A previous study showed that PIK3CA-mediated breast cancer cell growth and survival are dependent on the SGK3, and Akt is dispensable [17]. SGK3 is a unique member of the SGK family because it contains an N-terminal PX domain. SGK3 binds selectively to PtdIns(3)P through its PX domain, which is required for targeting SGK3 to the endosome, where the Class III PI3K (also termed hVps34) phosphorylates PtdIns to generate a pool of PtdIns(3)P [18, 19]. VPS34-IN1, an hVps34 inhibitor can suppress SGK3 activation by reducing PtdIns(3)P levels via lowering phosphorylation of T-loop and hydrophobic motifs [20, 21]. Amplification and overexpression of SGK3 have been reported more frequently than those for AKT in HCC, suggesting it may have a greater functional significance in HCC [22]. Our previous study found that SGK3 plays an important role in the invasive potential of HCC cells and epithelial-mesenchymal transition (EMT) [23]. However, the role and mechanism of SGK3 in CSCs has not been reported.

Here, we show that SGK3 is preferentially activated in liver CSCs, and upregulated or downregulated SGK3 in HCC cells enhances or suppresses liver CSC-associated gene expression and spheroid formation via the GSK-3 $\beta$ / $\beta$-catenin signalling pathway. We also found that prolonged treatment of HCC cells with PI3K inhibitors leads to activation of SGK3 and expansion of liver CSCs. Additionally, our results demonstrate that inhibition of
hVps34 can block SGK3 activity and suppress liver CSC expansion. Effective inhibition of SGK3 signalling may be useful in eliminating liver CSCs.

\section{Methods \\ Cell lines and culture}

The Huh7 cells were obtained from the Chinese Academy of Sciences Cell Bank. The MHCC-97H cells were obtained from Zhongshan Hospital of Fudan University in Shanghai, China. All cells were maintained in Dulbecco's modified Eagle medium (DMEM; Heclone) supplemented with $10 \%$ foetal bovine serum (FBS; Capricorn). Liver CSCs were cultured in serum-free culture medium. Serum-free culture medium was DMEM/F12 (Hyclone) consisting of $20 \mathrm{ng} / \mathrm{ml}$ basic fibroblast growth factor (bFGF; PeproTech), $20 \mathrm{ng} / \mathrm{ml}$ epidermal growth factor (EGF; PeproTech) and $20 \mu \mathrm{l} / \mathrm{ml}$ B27 supplement (Life Technologies).

\section{Isolation of CD133+ cells}

CD133+ cells were obtained using the CD133 MicroBead Kit (Miltenyi Biotec) according to the manufacturer's instructions. HCC cells were enzymatically dissociated, the cell suspension was centrifuged at $300 \times \mathrm{g}$ for $10 \mathrm{~min}$, and the supernatant was aspirated completely. The cells were resuspended in $300 \mu \mathrm{l}$ of buffer per $10^{8}$ cells, and $100 \mu \mathrm{l}$ of FcR Blocking Reagent and $100 \mu \mathrm{l}$ of CD133 MicroBeads were added, mixed well and incubated for $30 \mathrm{~min}$ at $4{ }^{\circ} \mathrm{C}$. Then, the cells were washed by adding $1 \mathrm{ml}$ of buffer, centrifuged at $300 \times \mathrm{g}$ for $10 \mathrm{~min}$, and sorted with the Mini MACS ${ }^{\bullet}$ Separator (Miltenyi Biotec). Phycoerythrin (PE)-conjugated CD133/2 antibodies (Miltenyi Biotec) were used to evaluate the efficiency of magnetic separation by flow cytometry.

\section{RNA extraction and quantitative reverse transcription polymerase chain reaction (RT-PCR)}

Total RNA was extracted from the cells by using Trizol (Invitrogen). Complementary DNA (cDNA) synthesis was performed using the PrimeScript ${ }^{\text {Tut }}$ RT Reagent Kit with gDNA Eraser (Takara). Quantitative RT-PCR was performed using the SYBR Premix ExTaq (Takara) under standard conditions according to the manufacturer's instructions. Quantitative RT-PCR was conducted with the CFX96 Real-Time PCR Detection System (Bio-Rad). The data were analysed using the $2^{-\triangle \Delta C t}$ method. The primers are listed in Additional file 1: Table S1.

\section{Cell siRNA transfection and inhibitors}

Small interfering RNAs (siRNA) of SGK3 and negative control (NC) were designed and synthesised by RiboBio (Guangzhou, China). The cells were transfected using a ribo $\mathrm{FECT}^{\mathrm{Tm}} \mathrm{CP}$ Transfection Kit (RiboBio) according to the manufacturer's protocol. A total of $2 \times 10^{5}$ cells were 
seeded per well and grown to $50-70 \%$ confluence. Transfection complexes were prepared according to the instructions and were added directly to the cells. The siRNA and NC were used at a final concentration of $100 \mathrm{nM}$. The mRNA level was detected by RT-PCR after incubation for $24 \mathrm{~h}$, and the level of protein was determined by western blot after incubation for $48 \mathrm{~h}$. Class I PI3K inhibitors ZSTK474 and LY294002, hVps34 inhibitor VPS34-IN1 and GSK $3 \beta$ inhibitor AR014418 were purchased from MedChem Express (MCE).

\section{Establishment of the SGK3 stable overexpression and knockdown cell lines}

To establish stable transduction, lentiviral vectors expressing SGK3 sequence, shRNA and the control vectors were obtained from Hanbio (Shanghai, China). shRNA-mediated silencing of SGK3 required the synthesis of a set of oligonucleotides composed of a target shRNA sequence and its complement against SGK3, as previously described [24]. Polybrene (Hanbio) was used to promote the transfection according to the manufacturer's instruction. On the previous day, $1 \times 10^{5}$ cells were seeded per well and grown to $20-40 \%$ confluence. Then, the cells were transfected at a multiplicity of infection (MOI) of 20. After $72 \mathrm{~h}$, the transfection efficiency was verified by fluorescence microscopy and RT-PCR.

\section{Spheroid formation assay}

The cell spheroid formation assay was performed as described previously [10]. Briefly, single cells $\left(1 \times 10^{3}\right)$ were plated in a 6-well ultra-low attachment plate (Corning) or $\left(1 \times 10^{2}\right)$ in a 24-well ultra-low attachment plate in the serum-free culture medium. After 1-2 weeks, the number of tumour spheroids (diameter $>50 \mu \mathrm{m}$ ) was counted under an inverted microscope.

\section{Protein extraction and western blotting}

Western blot analysis to determine protein level was performed as described previously [10]. The following antibodies were used: anti-SGK3 (sc-166,847; Santa Cruz), anti- $\beta$-actin (YT0099; Immunoway), anti-Akt1 (ab32505; Abcam), anti-Akt1 (phosphoS473; ab81283; Abcam), anti-SGK3 (phosphoThr320; \#5642; CST), anti-GSK3ß (ab32391, Abcam), anti-GSK3 $\beta$ (phosphoS9; ab75814; Abcam), anti- $\beta$-catenin (\#8480, CST), anti-CD133 (YT5192; Immunoway) and anti-Nanog (YM0464; Immunoway).

\section{Immunofluorescence}

Cell slides were fixed with $4 \%$ paraformaldehyde for $20 \mathrm{~min}$, and permeabilised with $0.3 \%$ Triton X-100 (Sigma-Aldrich) for $15 \mathrm{~min}$. Then, the cells were blocked with normal goat serum and incubated with anti-Nanog (1:100; Immunoway) at $4{ }^{\circ} \mathrm{C}$ overnight, followed by incubated with Alex 555-conjugated goat anti-mouse antibody for $1 \mathrm{~h}$. The cells were counterstained with $4^{\prime}$, 6-diamidino-2-phenylindole (DAPI) for $5 \mathrm{~min}$ and visualised by fluorescent microscope (Nikon).

\section{Immunohistochemical assay}

Tumour tissues from the nude mice were fixed in $4 \%$ formaldehyde for $24 \mathrm{~h}$, embedded in paraffin, and serially sectioned at a thickness of $6 \mu \mathrm{m}$. Sections were deparaffinized and stained with anti-CD133 (1:500; ab222782; Abcam) at $4{ }^{\circ} \mathrm{C}$ overnight, and incubated with the secondary antibody for $1 \mathrm{~h}$ at $37^{\circ} \mathrm{C}$. Reaction results were shown by incubation with $3,3^{\prime}$-Diaminobenzidine (DAB; Boster). After washing with tap water to stop the chromogenic reaction, the sections were dehydrated in an ascending alcohol gradient, cleared twice with xylene and mounted in neutral balsam. Then, the sections were examined and imaged by microscope (Nikon).

\section{Flow cytometric analysis}

Cells were collected and resuspended in $100 \mu \mathrm{l}$ of phosphate-buffered saline (PBS) containing $20 \mu \mathrm{l}$ FcR Blocking Reagent (Miltenyi Biotec) and anti-human antibodies, PE-CD133 (Miltenyi Biotec), and then incubated for $15 \mathrm{~min}$ on ice in the dark. After incubating, the cells were washed twice with $1 \mathrm{ml}$ of PBS. The collected cells were resuspended in $300 \mu \mathrm{l}$ of PBS and detected using a FACSCanto II flow cytometer (BD Biosciences). Isotype-matched mouse antibodies served as controls.

\section{In vivo xenograft experiments}

All animal experiments were performed in compliance with the strict rules of the Animal Ethics Committee of Chongqing Medical University. For tumour formation assay, $1 \times 10^{4}$ CD133+/- cells were subcutaneously injected into 6-week-old female athymic nude mice. Tumour formation was observed every week and analysed at the sixth week. The effect of ZSTK474 was tested in vivo. MHCC97H cells were subcutaneously injected into 6 -week-old female athymic nude mice $\left(5 \times 10^{6}\right.$ cells per mouse) and allowed to form tumours. Once the tumours reached $300 \mathrm{~mm}^{3}, 6$ animals were randomly divided into control and ZSTK474 groups. The ZSTK474 group was orally administered a dose of ZSTK474 (suspended in $5 \%$ hydroxypropyl cellulose) at $400 \mathrm{mg} / \mathrm{kg}$ daily for 10 days. The control group of mice was orally administered with 5\% hydroxypropyl cellulose instead of ZSTK474. Tumours were measured throughout the treatment period.

\section{Statistical analysis}

Statistical analyses were performed using SPSS 21 software. All data were acquired from at least 3 independent experiments and are reported as the mean \pm SD. Two 
independent group comparisons were analysed using Student's t-test. $P<0.05$ was considered statistically significant.

\section{Results}

SGK3 is preferentially activated in liver CSCs

It has been confirmed that the liver cancer cell spheroids cultured in serum-free culture medium are highly enriched in liver CSCs. To explore the relevance between SGK3 and liver CSCs, we detected expression levels of SGK3 mRNA in CSC-enriched hepatoma spheroids compared with monolayer-attached cells. The results showed that the mRNAs of several stem cell markers, including CD90, CD133, Oct4, Nanog, Bmi-1, and SOX2 were all upregulated in the spheroids compared to the monolayer-attached cells, and moderately elevated expression of SGK3 mRNA was observed in the spheroids (Fig. 1a). We next analysed SGK3 protein levels and phosphorylated levels in spheroids, and we found elevated SGK3 phosphorylation in spheroids, while no difference was observed between the expression of the total SGK3 protein in spheroids and attached cells. Consistently, the phosphorylation of Akt rather than total Akt proteins was elevated in spheroids (Fig. 1b).

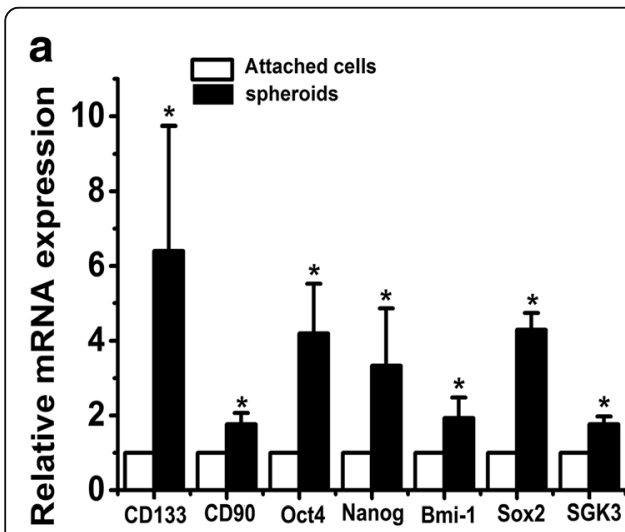

d

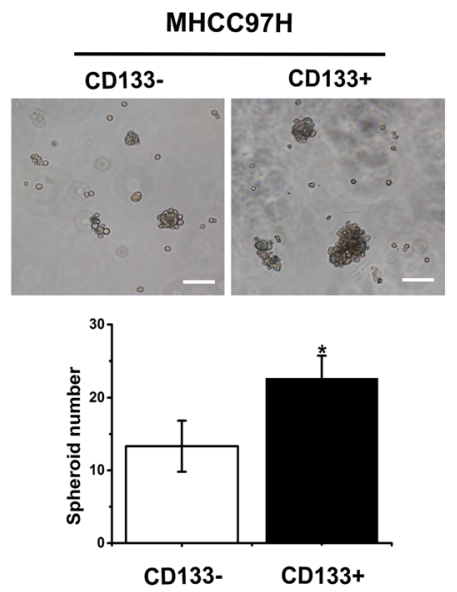

\section{b}

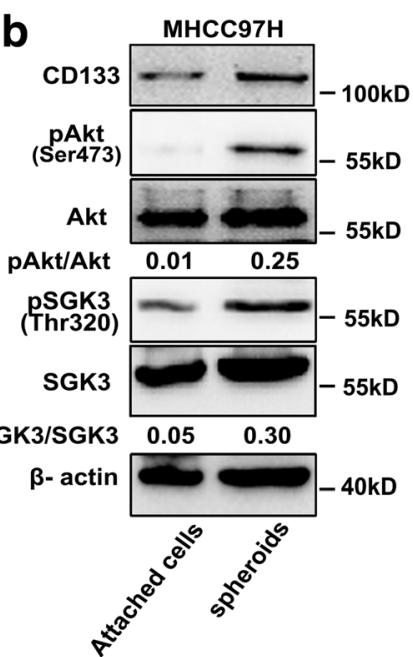

e

\begin{tabular}{lcc}
\multicolumn{3}{l}{ The tumor formation capacity of CD133- and CD133+ cells } \\
\hline Cells & $\begin{array}{l}\text { Number of } \\
\text { injected cells }\end{array}$ & $\begin{array}{l}\text { Number of mice } \\
\text { with tumor formation }\end{array}$ \\
\hline CD133- & $5 \times 10^{4}$ & $1 / 3$ \\
CD133+ & $5 \times 10^{4}$ & $3 / 3$ \\
\hline
\end{tabular}

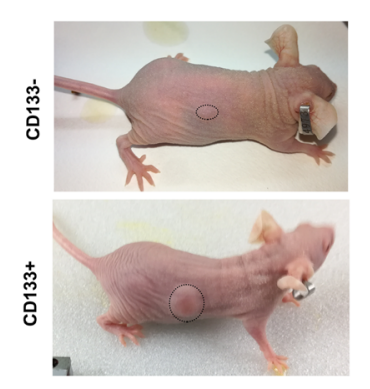

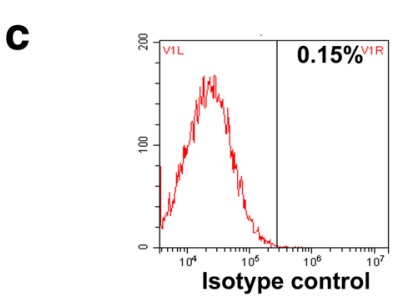

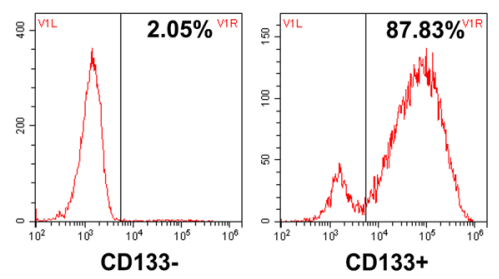

f

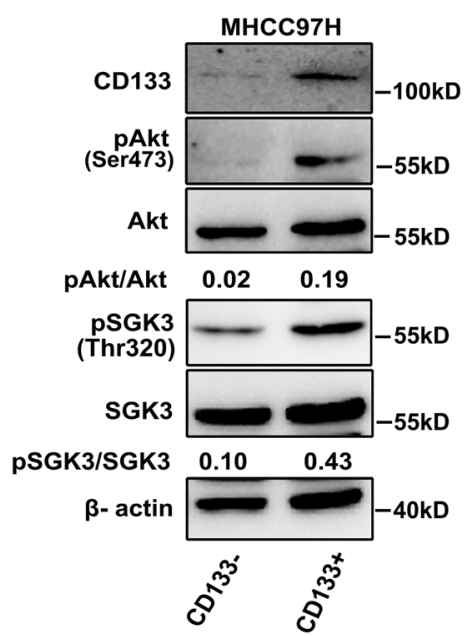

Fig. 1 SGK3 is preferentially activated in liver CSCs. a MHCC-97H cells were cultured in monolayer or ultra-low attachment conditions. The mRNA expression of liver CSC-related genes and SGK3 in spheroids and attached cells was compared by RT-PCR. $\mathbf{b}$ Western blot analysis for levels of CD133, active Akt (phosphorylated at Ser473)/total Akt and active SGK3 (phosphorylated at Thr320)/total SGK3 between spheroids and attached cells. c Flow cytometry analysis of CD133+ cell distribution in CD133- and CD133+ cells isolated using CD133 MicroBead Kit. d Representative images of CD133+ and CD133- cells sorted from MHCC97H HCC cells cultured in serum-free culture medium after 7 days. Scale bars, $100 \mu$ m. e CD133+/CD133- cells were subcutaneously injected into 6-week-old female athymic nude mice, and tumourigenicity was evaluated 5 weeks post-inoculation. $\mathbf{f}$ Levels of CD133, active Akt (phosphorylated at Ser473)/total Akt and active SGK3 (phosphorylated at Thr320)/total SGK3 were compared by western blot analysis between CD133+ and CD133- cells. $\beta$-actin was used as a loading control. All experiments were performed in triplicate. ${ }^{*} P<0.05$ 
CD133 is widely used as liver CSCs markers [25]. We then sorted CD133+ cells from MHCC97H HCC cells. After sorting, the purity of the selected CD133+ population was monitored by flow-cytometric analysis to ensure the positivity of CD133 was over 85\% (Fig. 1c). After cultured in serum-free culture medium for 7 days, the number of spheroids (diameter $>50 \mu \mathrm{m}$ ) in the CD133+ cells was significantly higher than that in the CD133- cells (Fig. 1d). Furthermore, CD133+ cells displayed much higher tumour-initiating capacity in in vivo experiments (Fig. 1e). To further investigate the role of SGK3 in liver CSCs, we determined the phosphorylation of SGK3 in CD133+ and CD133- cells. As expected, phosphorylation of SGK3 in CD133+ HCC cells was significantly higher than that in CD133- cells (Fig. 1f). In addition, the phosphorylation of Akt rather than total Akt proteins was elevated in CD133+ HCC cells (Fig. 1f), which is consistent with findings reported by Ma et al. [26]. Taken together, these results indicate that SGK3 may play a crucial role in the expansion of liver CSCs.

\section{SGK3 enhances the expansion of liver CSCs}

To assess the role of SGK3 in liver CSC regulation, we established a lentivirus-mediated stable SGK3 or negative control (NC) expression cell lines using MHCC-97H and Huh7. Notably, SGK3 overexpression significantly enhanced the mRNA expression of CSC-related genes CD133, CD90, Oct4, Nanog, Bmi-1 and Sox 2 compared with NC cells (Fig. 2a and b). Consistently, the expression of CD133 and Nanog proteins increased with the increase of SGK3 (Fig. 2c). To further elucidate the role of SGK3 in CSCs, we examined spheroid-forming ability (a surrogate marker of CSCs self-renewal). We found that SGK3 overexpression increased spheroid formation ability compared with NC cells (Fig. 2d). These results suggest that SGK3 enhances the expansion of liver CSCs.

\section{Inhibition of SGK3 attenuates liver CSCs expansion}

To further confirm the involvement of SGK3 in liver CSC expansion, 2 lentivirus vectors were designed to express shRNA for SGK3 knockdown (SGK3 shRNA1 and SGK3 shRNA2). After SGK3 inhibition, stemness genes were significantly suppressed in HCC cell lines (Fig. 3a and b). The protein levels of CD133 and Nanog were decreased in HCC cells after knockdown of SGK3, determined by western blot (Fig. 3c). In addition, SGK3 inhibition resulted in smaller spheroids, and they were significantly decreased in numbers (Fig. 3d). To confirm the result, flow cytometric analysis revealed a diminished proportion of liver CSCs in SGK3 shRNA1 stably transfected HCC cells (Fig. 3e).

\section{Prolonged treatment of HCC cells with PI3K inhibitors} leads to activation of SGK3 and expansion of liver CSCs Because SGK3 can be activated by PI3K independent of AKT, we tested whether the phosphorylation of SGK3 can be blocked by PI3K inhibitors. We treated Huh7 cells with PI3K inhibitors (LY294002 and ZSTK474) for $24 \mathrm{~h}$ with increasing doses. Interestingly, the results showed that both inhibitors induced a dose-dependent increase in SGK3 phosphorylation, while the phosphorylation of Akt was inhibited with increasing concentration (Fig. 4a). In addition, after PI3K inhibitor treatment, we observed a dose-dependent increase in the expression of CSC-relative genes CD133 and Nanog (Fig. 4a, b and c). A recent study reported that prolonged treatment of breast cancer cells with class I PI3K or Akt inhibitors leads to increased expression and activation of SGK3 [20]. To investigate whether prolonged treatment of liver cancer cells with PI3K inhibitors could promote expression and activation of SGK3, we treated cells with ZSTK474 in a time gradient $(0 \mathrm{~h}, 4 \mathrm{~h}, 8 \mathrm{~h}, 24 \mathrm{~h}, 48 \mathrm{~h}$, and $72 \mathrm{~h})$. As expected, prolonged ZSTK474 treatment over 24 to $72 \mathrm{~h}$ led to a time-dependent increase of SGK3 phosphorylation under conditions in which the CSC-relative genes CD133 and Nanog were induced (Fig. 4d).

Flow cytometric analysis revealed an enlarged proportion of CD133+ cells after Huh7 cells were treated with ZSTK474 for $72 \mathrm{~h}$ (Fig. 4e). Interestingly, knockdown of SGK3 expression employing siRNA partially blocked prolonged PI3K inhibitor treatment from enhancing CD133 expression in both Huh7 and MHCC97H cells (Fig. 4f). These results indicate that prolonged treatment with PI3K inhibitors induced expansion of CD133+ cells via enhancing SGK3 phosphorylation.

We next assessed the effect of ZSTK474 on tumour growth in an in vivo experiment. After the tumours formed $\left(300-500 \mathrm{~mm}^{3}\right)$, the mice were orally administered with $400 \mathrm{mg} / \mathrm{kg}$ of ZSTK474 for 10 days. The results indicate that ZSTK474 showed significant anti-tumour activity in the treatment period as compared to control mice (Fig. 4g). Western blot analysis of ZSTK474-treated tumours exhibited increased expression of Nanog and CD133, and the increased phosphorylation of SGK3 was validated (Fig. 4h). The increased mRNA level of CD133 was also confirmed in vivo (Fig. $4 \mathrm{i})$. Immunohistochemical assay showed that tumours treated with ZSTK474 have higher levels of CD133 than control tumours (Fig. 4j).

\section{The inhibitor of hVps34 can block SGK3 activity and suppress liver CSC expansion induced by PI3K inhibitors} It has been reported that SGK3 can be activated by PtdIns(3)P produced by hVps34, and hVps34 inhibitor VPS34-IN1 can inhibit SGK3 activation [20, 21]. Our 
a

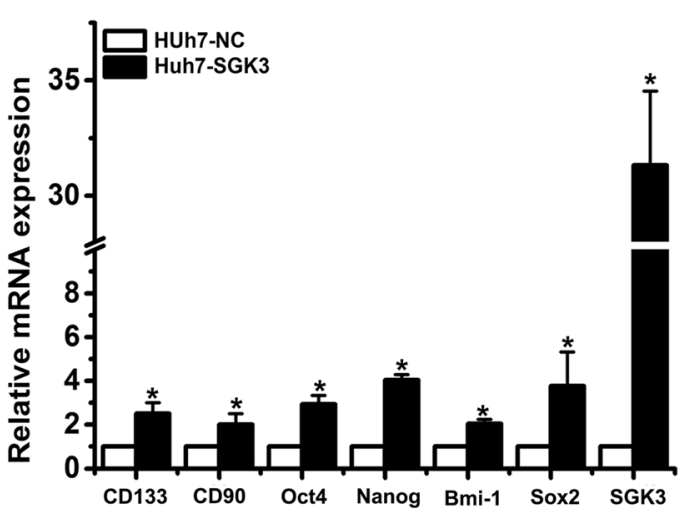

C

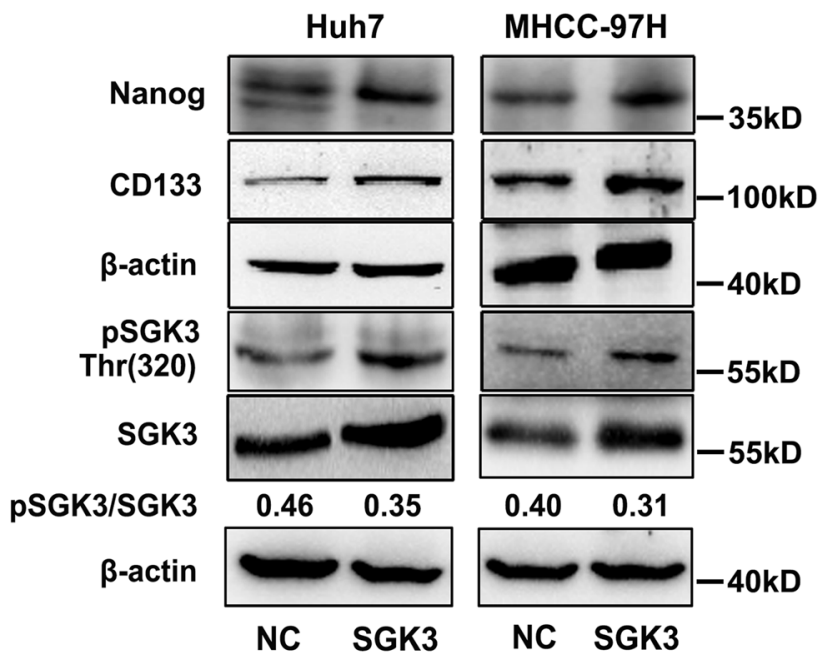

b $\quad 21] \square$ MHCC97H-NC

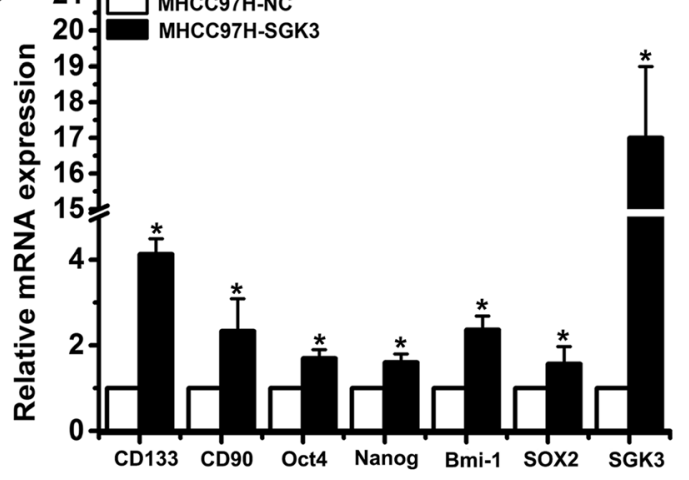

d

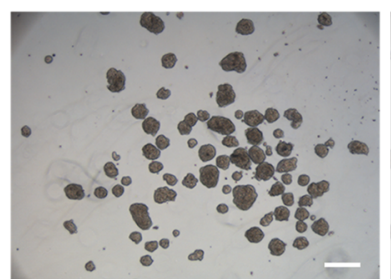

NC

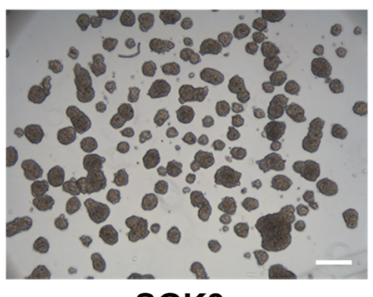

SGK3

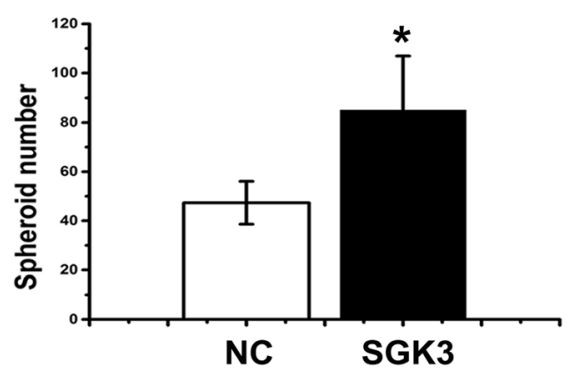

Fig. 2 SGK3 enhances the expansion of liver CSCs. a, b Relative mRNA expression of liver CSC-related markers CD133, CD90, Nanog, Oct4, Bmi-1 and Sox2 in Huh7 and MHCC-97H cells stably overexpressing SGK3 or NC. $\beta$-actin was used as an loading control. c Expression of CD133, Nanog and SGK3 in Huh7 and MHCC-97H cells stably overexpressing SGK3 or NC detected by western blot. $\beta$-actin was used as a loading control. d Spheroid formation assay of MHCC97H-SGK3 or MHCC97H-NC cells (top). Scale bars, $200 \mu \mathrm{m}$. The statistical results of the tumour spherosis (> $50 \mu \mathrm{m}$ ) were calculated based on 3 independent experiments (bottom). All experiments were performed in triplicate, and the results are shown as mean \pm standard deviation. ${ }^{*} P<0.05$

results confirmed that VPS34-IN1 induced a dosedependent inhibition of SGK3 phosphorylation in MHH97H and Huh7 cells (Fig. 5a). In addition, treatment of Huh7 and MHCC97H cells with VPS34-IN1 also reduced the expression of CSC-related markers CD133 and Nanog (Fig. 5a and c). Consistently, hVps34 inhibitor VPS34-IN1 suppressed the spheroid formation ability tested by spheroid formation assay (Fig. 5b). Flow cytometric analysis revealed a diminished proportion of CD133+ cells after Huh7 cells were treated with VPS34-IN1 for $24 \mathrm{~h}$ (Fig. 5d). Furthermore, the increased expression of CD133 and Nanog induced by prolonged PI3K inhibitor treatment can be depleted by VPS34-IN1 (Fig. 5e). Collectively, these results indicated that the inhibitor of hVps34 can block liver CSC expansion after prolonged treatment of HCC cells with PI3K inhibitors via the inhibition of SGK3.

\section{SGK3 promotes liver CSCs through $\beta$-catenin accumulation} by GSK3 $\beta$

Our previous study confirmed that SGK3 stimulates $\beta$-catenin signalling in HCC cells [23]. It is well established that SGK3 promotes the inactivation of GSK-3 $\beta$ by phosphorylation of GSK-3 $\beta$ on Ser9 [22]. To investigate whether SGK3 can promote liver CSC expansion via GSK-3 $\beta / \beta$-catenin signalling, we first detected the expression of GSK-3 $\beta$ and $\beta$-catenin in spheroids and attached cells. The western blot analysis revealed that the phosphorylation of GSK-3 $\beta$ on Ser9 and expression of $\beta$-catenin was significantly increased in spheroid 


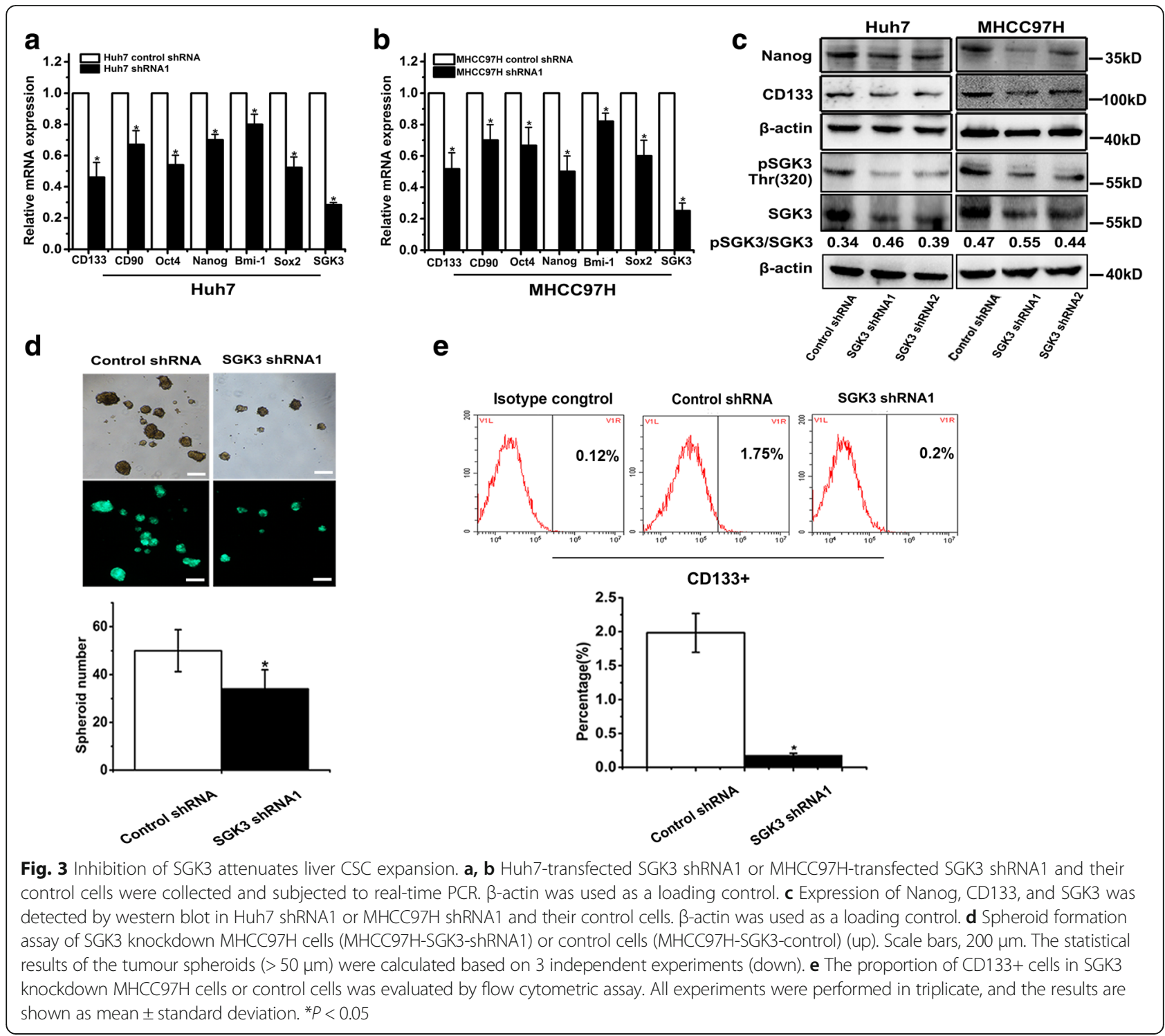

cells (Fig. 6a). We next analysed the phosphorylation of GSK-3 $\beta$ on Ser9 after SGK3 overexpression or knockdown. Our results indicated that overexpression of SGK3 increased the phosphorylation level of GSK-3 $\beta$ on Ser9 (Fig. 6b). Conversely, knockdown of SGK3 reduced the phosphorylation level of GSK-3 $\beta$ on Ser9 (Fig. 6c). Furthermore, overexpression of SGK3 increased $\beta$-catenin levels, whereas knockdown of SGK3 reduced $\beta$-catenin expression (Fig. $6 \mathrm{~b}$ and c), indicating a canonical regulation of $\beta$-catenin stability by SGK3. Treatment of MHCC97H cells with AR-A014418, a selective GSK-3 $\beta$ inhibitor reduced the expression of CD133 (Fig. 6d). To further determine the role of SGK3 in liver CSC self-renewal via the GSK-3 $\beta / \beta$-catenin signalling pathway, SGK3 overexpression and control cells were treated with AR-A014418. Spheroid formation analysis showed that AR-A014418 weakened the SGK3-enhanced self-renewal of liver CSCs (Fig. 6e).

\section{Prolonged treatment of HCC cells with PI3K inhibitors} stimulates the $\beta$-catenin signalling pathway via activation of SGK3

Nuclear accumulation of $\beta$-catenin has been reported to confer resistance to PI3K inhibitors in colon cancer [27]. To explore the $\beta$-catenin levels changed under prolonged treatment of HCC cells with PI3K inhibitors, we treated MHCC97H cells with PI3K inhibitors for 0 to 5 days and detected $\beta$-catenin expression. The results showed that prolonged PI3K inhibitor ZSTK474 treatment over 1 to 5 days led to a time-dependent accumulation of $\beta$-catenin (Fig. 7a). To study the effect that PI3K inhibitors had on $\beta$-catenin accumulation via 


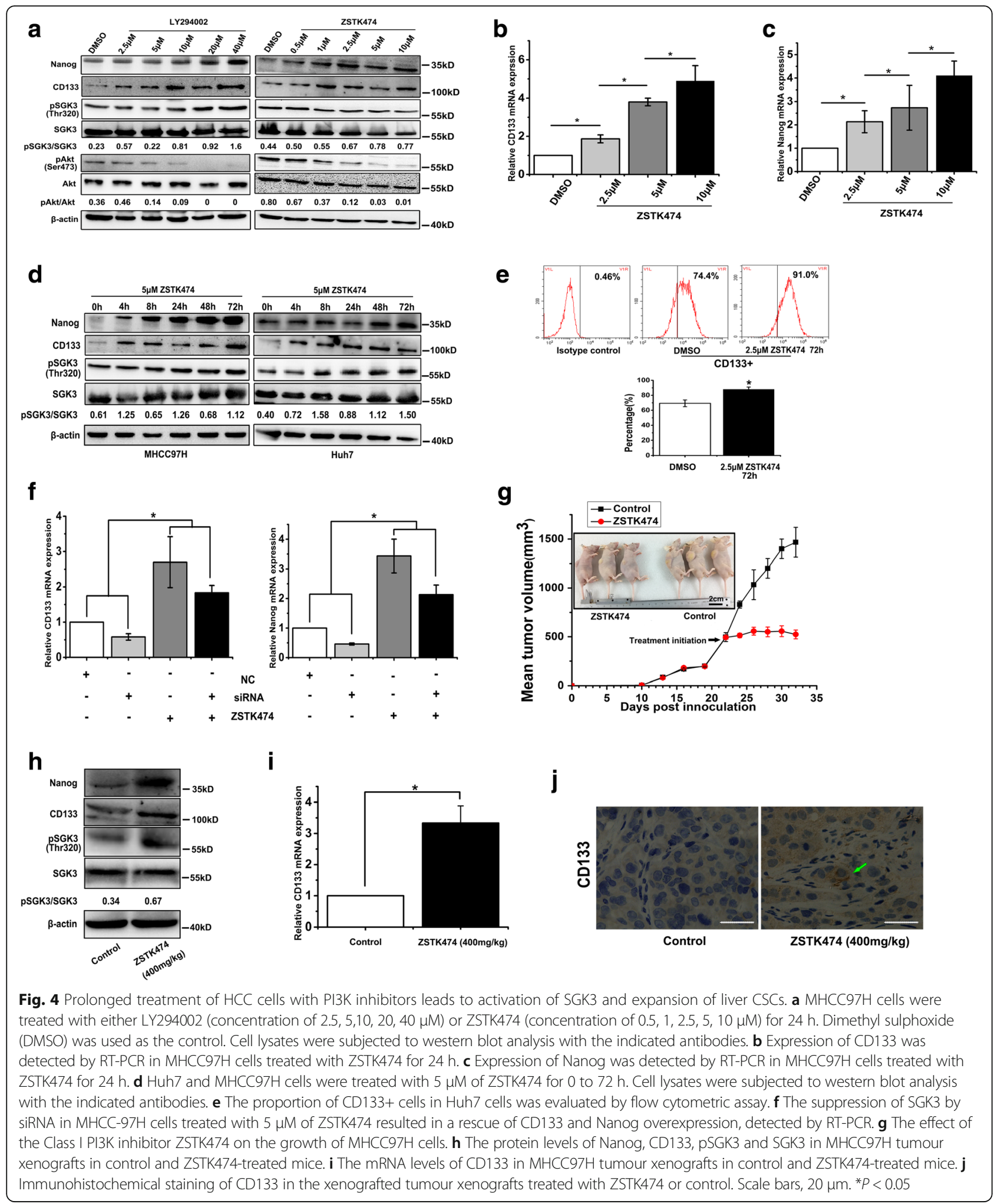

SGK3, SGK3 was knocked down in MHCC97H cells by siRNAs and subjected to treatment with ZSTK474. As expected, inhibition of SGK3 decreased the accumulation of $\beta$-catenin after treatment with ZSTK474 for $72 \mathrm{~h}$
(Fig. 7b). Taken together, these data suggest that prolonged treatment of HCC cells with inhibitors of PI3K leads to marked accumulation of $\beta$-catenin via the activation of SGK3 (Fig. 7c). 


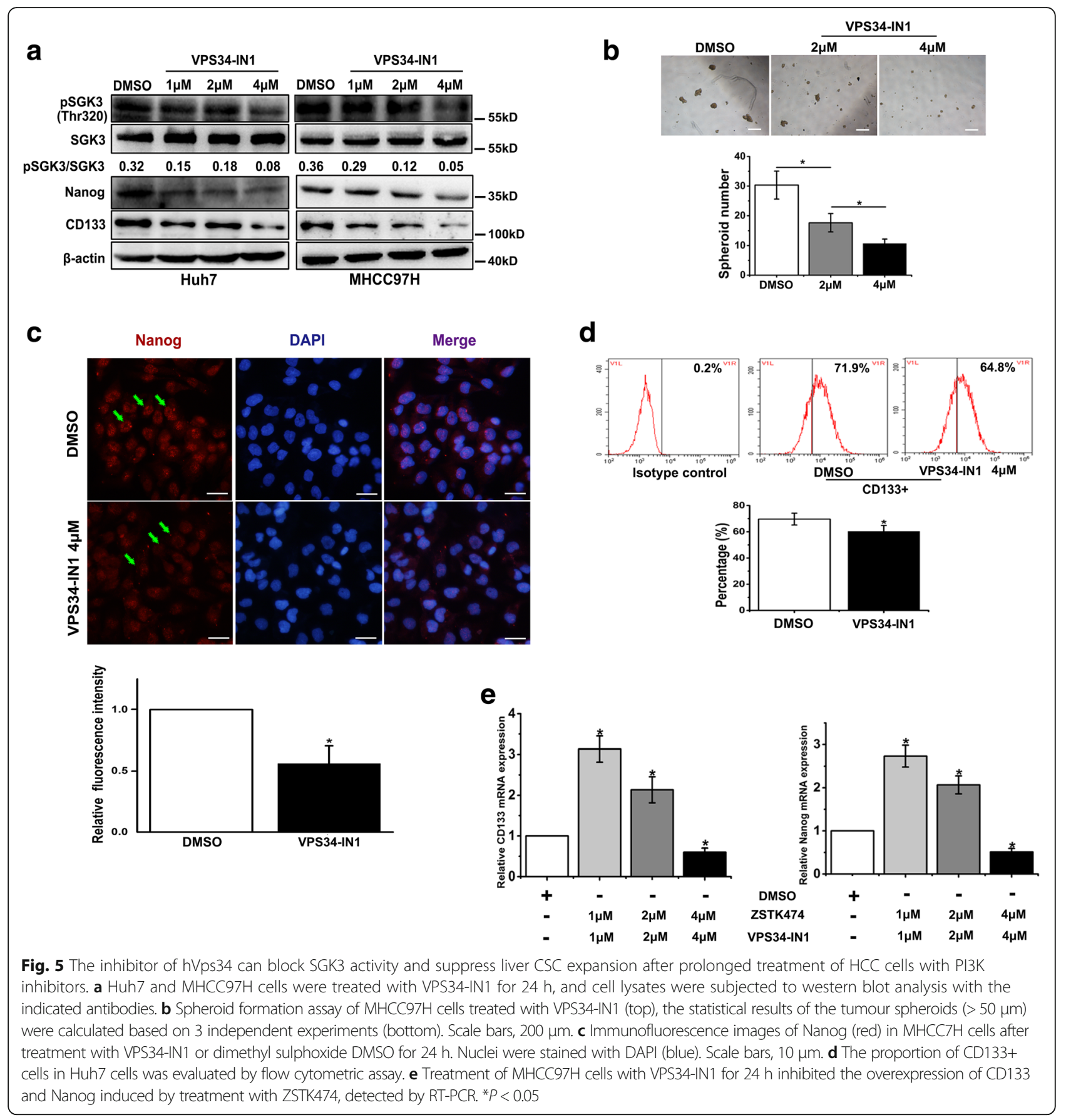

\section{Discussion}

CSCs have stem characteristics such as self-renewal, differentiation and tumourigenesis. CSCs have been recognised to contribute to cancer relapse and metastasis due to their invasive and drug-resistant capacities. It is therefore important to explore the molecular mechanism underlying liver CSC regulation so as to develop novel therapeutic strategies eliminating CSCs. In this study, we report that SGK3 plays a pivotal role in liver CSC expansion via the GSK3 $\beta / \beta$-catenin signalling pathway, and the activation of SGK3 may contribute to the liver CSCs tolerance of PI3K inhibitor treatment.

SGK3 is emerging as a tumour oncogene in several cancers [28, 29]. Amplification and overexpression of SGK3 was frequently detected in HCC specimens, and SGK3 can promote HCC cell survival, proliferation and tumour formation in nude mice [22]. Our previous study found that SGK3 promotes HCC cell migration and invasive potential [23], which confirmed its vital function in promoting $\mathrm{HCC}$ progression. However, whether 


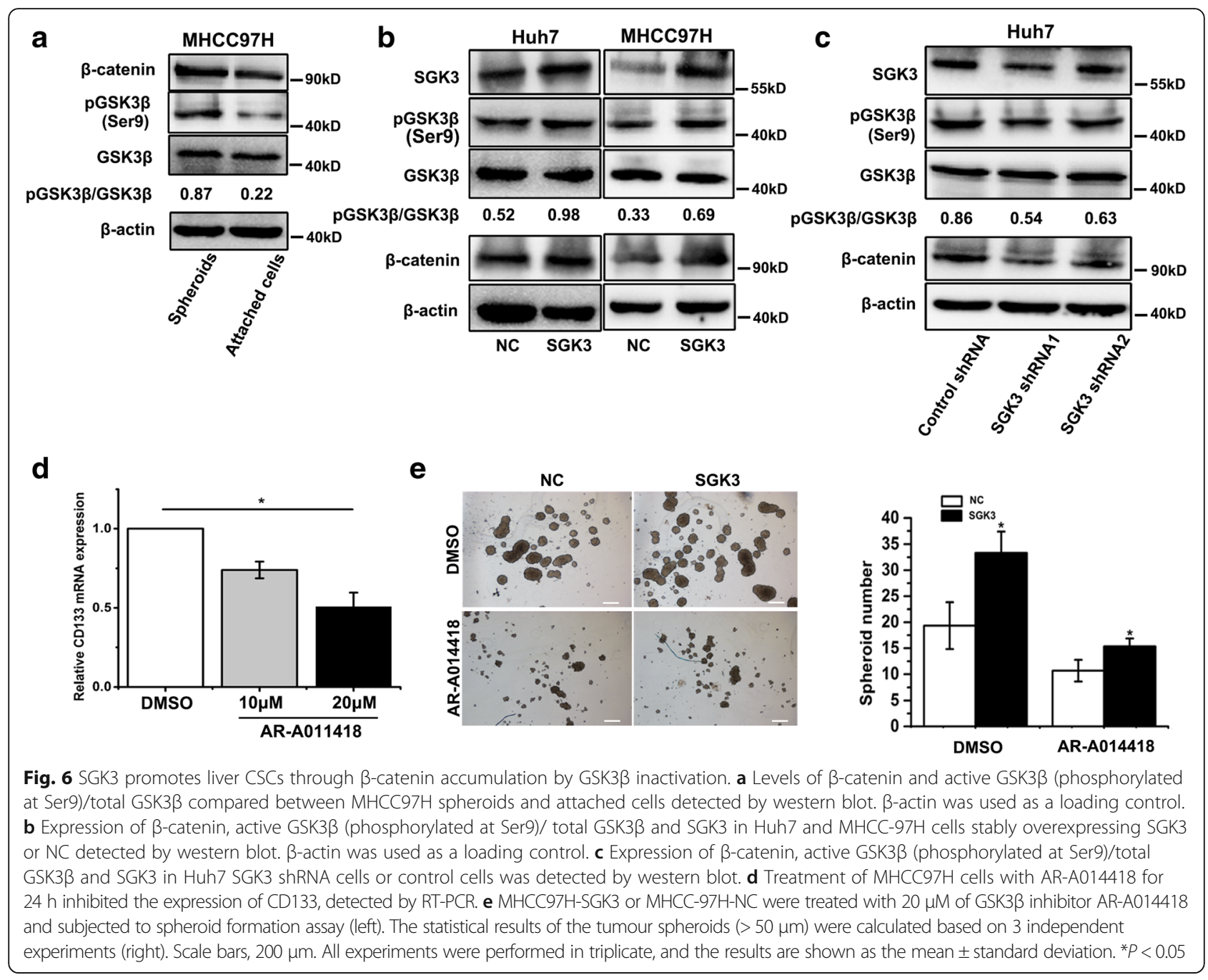

SGK3 also plays an important role in CSCs has not yet been reported. Our data showed a high phosphorylation level of SGK3 in liver CSC-enriched spheroids and CD133+ cells. More intriguingly, we demonstrated that overexpression of SGK3 enhances the expansion of liver CSCs, while inhibition of SGK3 by shRNA had an opposite effect. These findings indicate that SGK3 is critical for liver CSC expansion.

PI3K activity is stimulated by diverse oncogenes and growth factor receptors, and elevated PI3K signalling is considered a hallmark of cancer. Many PI3K pathway-targeted therapies have been tested in oncology trials [30]. Class I PI3K activates downstream effectors by generating phosphoinositides PtdIns-3, 4-P2 and PtdIns-3, 4, 5-P3. The shared property of these PI3K effectors is a pleckstrin homology $(\mathrm{PH})$ domain selective for PtdIns-3, 4, 5-P3 or PtdIns-3, 4-P2 [30, 31]. Arguably, the vast majority of studies have focused on the protein kinase AKT as the dominant effector of PI3K signalling associated with malignancy. SGK3 has been reported to be a critical effector of oncogenic PIK3CA mutant breast cancer cells in which Akt is dispensable [17]. Although SGK3 lacks a PH domain, it may still be activated by Class I PI3K through PDK1 [29]. In contrast to Akt, SGK3 possesses an N-terminal PtdIns(3)P-binding PX domain $[20,32]$, which is predominantly produced at the endosome by the Class III PI3K hVps34. In the present study, we demonstrated that prolonged treatment with PI3K inhibitors triggers SGK3 activation in HCC cells, while the phosphorylation of Akt is inhibited, which is consistent with findings reported by Bago et al. [20]. We reasoned that the activation of SGK3 may be a compensatory mechanism to substitute the blockade of the Akt signalling pathway, which causes HCC cells to tolerate the treatment of PI3K inhibitors.

In the present study, we also found that prolonged treatment with PI3K inhibitors induced expansion of liver CSCs in HCC cells. We speculated that the expansion of liver CSCs induced by prolonged treatment with PI3K inhibitors occurred via the activation of SGK3. To 


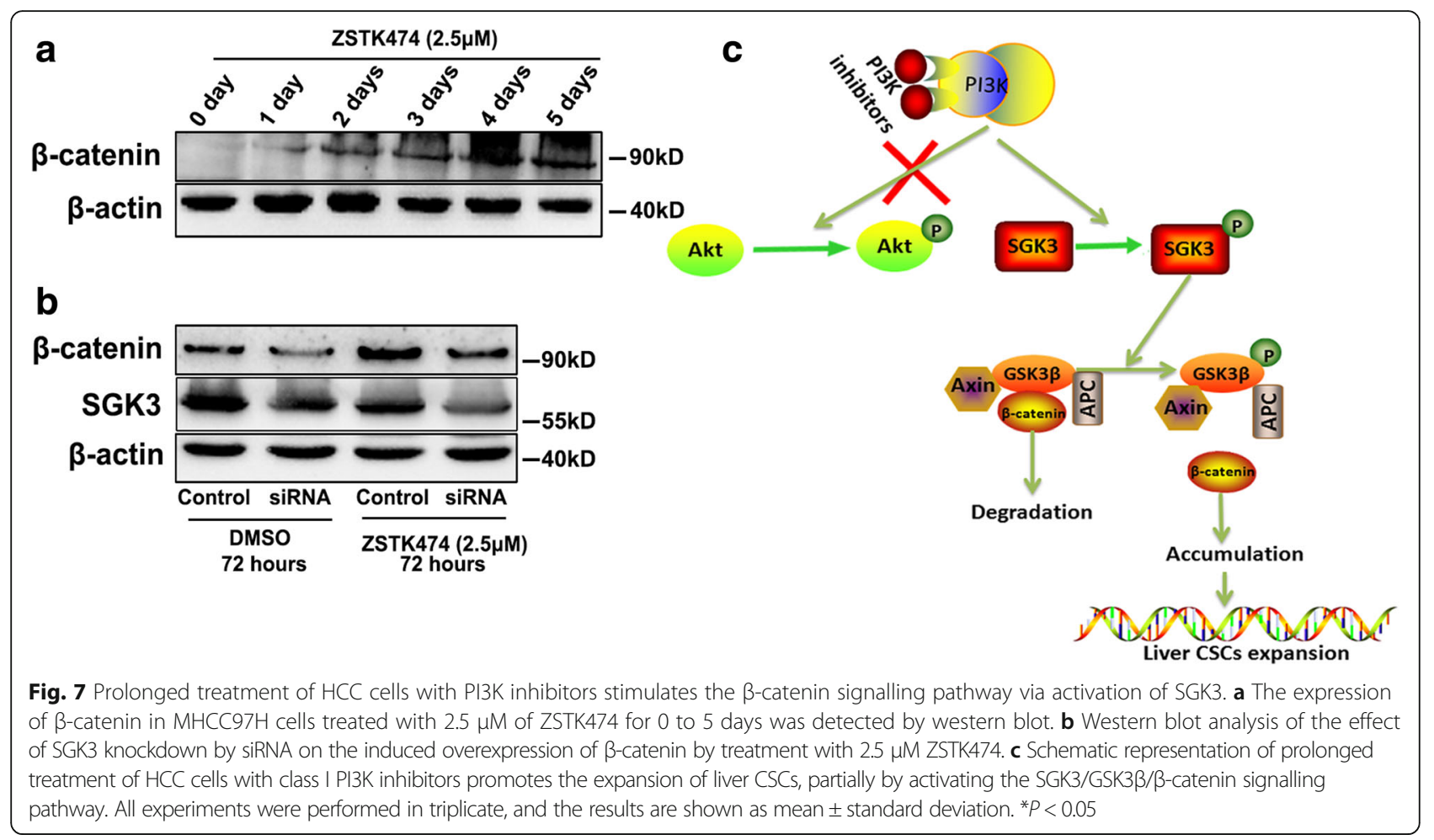

further confirm this hypothesis, a rescue experiment was performed using SGK3 siRNA. The results indicated that knockdown of SGK3 expression employing siRNA partially blocked prolonged PI3K inhibitor treatment from enhancing CD133 expression in both Huh7 and MHCC97H cells. Meanwhile, our in vivo models confirmed that decreased tumour growth would be achieved by ZSTK474 treatment. Consistent with the in vitro experiment, ZSTK474 treatment induced the expansion of liver CSCs and activation of SGK3 in vivo. Although ZSTK474 could inhibit the tumor growth via inhibit proliferation and promote apoptosis of non-stem cells, a small proportion of cells could survive via their owned or acquired stemness property. Inhibition of mTOR signalling has been reported to upregulate CD133 expression in gastrointestinal cancer cells [33]. Because the PI3K signalling pathway is a complex regulatory network, other downstream signal molecules may play a role in the expansion of liver CSCs induced by prolonged treatment with PI3K inhibitors. Class I PI3K is known to negatively regulate autophagy via the AKT-MTORC1-ULK1 complex [34]; thus, autophagy other than SGK3 may also be involved in regulating the expansion of liver CSCs after prolonged treatment with PI3K inhibitors in HCC cells.

By virtue of its PX domain, SGK3 can bind to PtdIns(3)P produced by hVps34 on the endosome; thus, hVps34 inhibitor can inhibit SGK3 activation. Our results confirmed that VPS34-IN1, an hVps34 inhibitor, induced a dose-dependent inhibition of SGK3 phosphorylation. Furthermore, the inhibition of hVps34 also causes the inhibition of liver CSC self-renewal. These results further confirm that SGK3 is critical for liver CSC expansion and that targeting SGK3 could be a promising strategy for HCC therapy.

The augmentation of $\mathrm{Wnt} / \beta$-catenin signalling through Ser9 phosphorylation-inactivation of GSK3 $\beta$ is a well-recognised regulatory pathway for CSC self-renewal and cancer development [35, 36]. Our previous study confirmed that SGK3 stimulates $\beta$-catenin signalling in HCC cells [23]. Liu et al. reported that overexpression of SGK3 increased the phosphorylation level of GSK3- $\beta$ on Ser9 and inactivates GSK3- $\beta$ [22]. In our present study, we further confirmed that SGK3 promotes $\beta$-catenin accumulation by increasing the phosphorylation level of GSK3- $\beta$ on Ser9, inactivating GSK3- $\beta$ and inhibiting the degradation of $\beta$-catenin. Importantly, our results demonstrated that SGK3 promotes liver CSC expansion through the GSK3 $\beta / \beta$-catenin signalling pathway. It has been previously reported that nuclear accumulation of $\beta$-catenin confers resistance to PI3K inhibitors in colon cancer [27]. Indeed, our data suggested that prolonged inhibition of Class I PI3K leads to significant accumulation of $\beta$-catenin. We speculated that the accumulation of $\beta$-catenin was through the activation of SGK3 in HCC cells subjected to prolonged inhibition of Class I PI3K, which led to the enhanced self-renewal of liver CSCs. 


\section{Conclusions}

In summary, our findings reveal that SGK3, a novel oncogene, plays a vital role in the expansion of liver CSCs through the GSK3 $\beta / \beta$-catenin signalling pathway. Prolonged treatment of HCC cells with class I PI3K inhibitors promotes the expansion of liver CSCs, partially by activating the SGK3/ GSK3 $\beta / \beta$-catenin signalling pathway (Fig. 7c). However, there is no specific inhibitor to SGK3. Perhaps, the development of this inhibitor will effectively eliminate the CSCs and improve the effect of PI3K inhibitors in the treatment of cancers.

\section{Additional file}

Additional file 1: Table S1. Primer sequences for quantitative RT-PCR (DOCX $24 \mathrm{~kb})$

\section{Abbreviations}

CSCs: Cancer stem cells; GSK3ß: Glycogen synthase kinase 33; HCC: Hepatocellular carcinoma; PI3K: Phosphoinositide 3-kinase; SGK3: Serum and glucocorticoidregulated kinase 3

\section{Funding}

This work was supported by the National Natural Science Foundation of China (81572888)

\section{Availability of data and materials}

The datasets used or analysed during the current study are available from the corresponding author upon reasonable request.

\section{Authors' contributions}

FCL designed and performed experiments, analysed data and wrote the paper; XLW designed experiments and analysed data; XJ performed experiments and analysed data; YZQ performed some experiments; JG initiated the study and organised, designed and wrote the paper. All authors read, discussed and approved the final manuscript.

\section{Ethics approval and consent to participate}

Not applicable.

\section{Consent for publication}

Not applicable.

\section{Competing interests}

The authors declare that they have no competing interests.

\section{Publisher's Note}

Springer Nature remains neutral with regard to jurisdictional claims in published maps and institutional affiliations.

Received: 28 December 2017 Accepted: 7 June 2018

Published online: 25 June 2018

\section{References}

1. Bruix J, Gores GJ, Mazzaferro V. Hepatocellular carcinoma: clinical frontiers and perspectives. Gut. 2014;63:844-55.

2. Chatterjee R, Mitra A. An overview of effective therapies and recent advances in biomarkers for chronic liver diseases and associated liver cancer. Int Immunopharmacol. 2015:24:335-45.

3. Forner A, Llovet JM, Bruix J. Hepatocellular carcinoma. Lancet. 2012;379: 1245-55.

4. Visvader JE. Cells of origin in cancer. Nature. 2011;469:314-22.

5. Easwaran H, Tsai HC, Baylin SB. Cancer epigenetics: tumor heterogeneity, plasticity of stem-like states, and drug resistance. Mol Cell. 2014;54:716-27.
6. Mitra A, Mishra L, Li SEMT. CTCS and CSCs in tumor relapse and drugresistance. Oncotarget. 2015;6:10697-711.

7. Koren E, Fuchs $Y$. The bad seed: cancer stem cells in tumor development and resistance. Drug Resist Updat. 2016;28:1-12.

8. Nio K, Yamashita T, Kaneko S. The evolving concept of liver cancer stem cells. Mol Cancer. 2017;16:4.

9. Chai S, Ng KY, Tong M, Lau EY, Lee TK, Chan KW, et al. Octamer 4/ microRNA-1246 signaling axis drives Wnt/B-catenin activation in liver cancer stem cells. Hepatology. 2016;64:2062-76.

10. Liu F, Kong $X$, Lv L, Gao J. TGF- $\beta 1$ acts through miR-155 to down-regulate TP53INP1 in promoting epithelial-mesenchymal transition and cancer stem cell phenotypes. Cancer Lett. 2015;359:288-98.

11. Mitra A, Yan J, Xia X, Zhou S, Chen J, Mishra L, Li S. IL6-mediated inflammatory loop reprograms normal to epithelial-mesenchymal transition + metastatic cancer stem cells in preneoplastic liver of transforming growth factor beta-deficient $\beta 2$-spectrin+/- mice. Hepatology. 2017;64:1222-36.

12. Tong M, Fung TM, Luk ST, Ng KY, Lee TK, Lin CH, et al. ANXA3/JNK signaling promotes self-renewal and tumor growth, and its blockade provides a therapeutic target for hepatocellular carcinoma. Stem Cell Rep. 2015;5:45-59.

13. Holland JD, Klaus A, Garratt AN, Birchmeier W. Wht signaling in stem and cancer stem cells. Curr Opin Cell Biol. 2013;25:254-64.

14. Janku F. Phosphoinositide 3-kinase (PI3K) pathway inhibitors in solid tumors: from laboratory to patients. Cancer Treat Rev. 2017;59:93-101.

15. Xia $P, X u X Y$. PI3K/Akt/mTOR signaling pathway in cancer stem cells: from basic research to clinical application. Am J Cancer Res. 2015;5:1602-9.

16. Bruhn MA, Pearson RB, Hannan RD, Sheppard KE. AKT-independent PI3K signaling in cancer-emerging role for SGK3. Cancer Manag Res. 2013;5:281-92.

17. Vasudevan KM, Barbie DA, Davies MA, Rabinovsky R, MCNear CJ, Kim JJ, et al. AKT-independent signaling downstream of oncogenic PIK3CA mutations in human cancer. Cancer Cell. 2009;16:21-32.

18. Ellson CD, Andrews S, Stephens LR, Hawkins PT. The PX domain: a new phosphoinositide-binding module. J Cell Sci. 2002;115:1099-105.

19. Virbasius JV, Song X, Pomerleau DP, Zhan Y, Zhou GW, Czech MP. Activation of the Akt-related cytokine-independent survival kinase requires interaction of its phox domain with endosomal phosphatidylinositol 3-phosphate. Proc Natl Acad Sci U S A. 2001;98:12908-13.

20. Bago R, Sommer E, Castel P, Crafter C, Bailey FP, Shpiro N, et al. The hVps34SGK3 pathway alleviates sustained PI3K/Akt inhibition by stimulating mTORC1 and tumour growth. EMBO J. 2016;35:1902-22.

21. Bago R, Malik N, Munson MJ, Prescott AR, Davies P, Sommer E, et al. Characterization of VPS34-IN1, a selective inhibitor of Vps34, reveals that the phosphatidylinositol 3-phosphate-binding SGK3 protein kinase is a downstream target of class III phosphoinositide 3-kinase. Biochem J. 2014;463:413-27.

22. Liu M, Chen L, Chan TH, Wang J, Li Y, Li Y, et al. Serum and glucocorticoid kinase 3 at 8q13.1 promotes cell proliferation and survival in hepatocellular carcinoma. Hepatology. 2012;55:1754-65.

23. Kong X, Liu F, Gao J. MiR-155 promotes epithelial-mesenchymal transition in hepatocellular carcinoma cells through the activation of PI3K/SGK3/ $\beta$ catenin signaling pathways. Oncotarget. 2016;7:66051-60.

24. Gasser JA, Inuzuka H, Lau AW, Wei W, Beroukhim R, Toker A. SGK3 mediates INPP4B-dependent PI3K signaling in breast cancer. Mol Cell. 2014;56:595-607.

25. Ma S, Chan KW, Hu L, Lee TK, Wo JY, Ng IO, et al. Identification and characterization of tumorigenic liver cancer stem/progenitor cells. Gastroenterology. 2007;132:2542-56.

26. Ma S, Lee TK, Zheng BJ, Chan KW. Guan XYCD133+ HCC cancer stem cells confer chemoresistance by preferential expression of the Akt/PKB survival pathway. Oncogene. 2008;27:1749-58.

27. Tenbaum SP, Ordonez-Moran P, Puig I, Chicote I, Arques O, Landolfi S, et al. $\beta$-catenin confers resistance to PI3K and AKT inhibitors and subverts FOXO3a to promote metastasis in colon cancer. Nat Med. 2012;18:892-901.

28. Guo ST, Chi MN, Yang RH, Guo XY, Zan LK, Wang CY, et al. INPP4B is an oncogenic regulator in human colon cancer. Oncogene. 2016;35:3049-61.

29. Scortegagna M, Lau E, Zhang T, Feng Y, Sereduk C, Yin H, et al. PDK1 and SGK3 contribute to the growth of BRAF-mutant melanomas and are potential therapeutic targets. Cancer Res. 2015;75:1399-412.

30. Fruman DA, Chiu H, Hopkins BD, Bagrodia S, Cantley LC, Abraham RT. The PI3K pathway in human disease. Cell. 2017;170:605-35.

31. Lien EC, Dibble CC, Toker A. PI3K signaling in cancer: beyond AKT. Curr Opin Cell Biol. 2017;45:62-71. 
32. Tessier M, Woodgett JR. Role of the Phox homology domain and phosphorylation in activation of serum and glucocorticoid-regulated kinase3. J Biol Chem. 2006;281:23978-89.

33. Matsumoto K, Arao T, Tanaka K, Kaneda H, Kudo K, Fujita Y, et al. mTOR signal and hypoxia-inducible factor-1a regulate CD133 expression in cancer cells. Cancer Res. 2009:69:7160-4.

34. Yu X, Long YC, Shen HM. Differential regulatory functions of three classes of phosphatidylinositol and phosphoinositide 3-kinases in autophagy. Autophagy. 2015;11:1711-28.

35. Chua HH, Tsuei DJ, Lee PH, Jeng YM, Lu J, Wu JF, et al. RBMY, a novel inhibitor of glycogen synthase kinase $3 \beta$, increases tumor stemness and predicts poor prognosis of hepatocellular carcinoma. Hepatology. 2015;62:1480-96.

36. Xiang D, Cheng Z, Liu H, Wang X, Han T, Sun W, et al. Shp2 promotes liver cancer stem cell expansion by augmenting $\beta$-catenin signaling and predicts chemotherapeutic response of patients. Hepatology. 2017;65:1566-80.

Ready to submit your research? Choose BMC and benefit from:

- fast, convenient online submission

- thorough peer review by experienced researchers in your field

- rapid publication on acceptance

- support for research data, including large and complex data types

- gold Open Access which fosters wider collaboration and increased citations

- maximum visibility for your research: over $100 \mathrm{M}$ website views per year

At BMC, research is always in progress.

Learn more biomedcentral.com/submissions 